\title{
Myeloid-derived suppressor cells in ovarian cancer: friend or foe?
}

\author{
MONIKA WALANKIEWICZ ${ }^{1,2}$, EWELINA GRYWALSKA ${ }^{1}$, GRZEGORZ POLAK', JAN KOTARSKI', \\ DOROTA J. SIWICKA-GIEROBA ${ }^{1,3}$, JACEK ROLINSSKI ${ }^{1}$
}

${ }^{1}$ Department of Clinical Immunology and Immunotherapy, Medical University of Lublin, Poland

${ }^{2}$ The First Department of Gynaecological Oncology and Gynaecology, Medical University in Lublin, Poland

${ }^{3}$ Department of Anaesthesiology and Intensive Care, Medical University of Lublin, Poland

\begin{abstract}
Although previous decades contributed to major progress in targeted therapy of many malignancies, the treatment of gynaecological cancers remains a challenging task. In the evidence of rising cancer mortality, the search for new methods of treatment is a dire need. Exploring the mechanisms of interaction between tumour cells and host immune response may allow the introduction of new, effective therapies - not as toxic and far more efficient than conventional methods of cancer treatment. Epithelial ovarian cancer (EOC) is typically diagnosed at advanced stages. Its incidence and mortality rate is high. Powerful diagnostic tools for this kind of cancer are still under investigation. Multiple mechanisms existing in the ovarian tumour network create a specific immunosuppressive microenvironment, in which accumulation of myeloid-derived suppressor cells (MDSCs) may be a critical component for diagnosis and treatment. This review attempts to verify current knowledge on the role of MDSCs in EOC.
\end{abstract}

Key words: ovarian cancer, myeloid-derived suppressor cells (MDSCs), T cells.

(Centr Eur J Immunol 2017; 42 (4): 383-389)

\section{Introduction}

Causes of impaired function of the immune system associated with cancer have been studied for many years, but their final explanation is still ahead of us. The anti-tumour response involves, among others, macrophages, NK cells, and cytotoxic T lymphocytes. Exploring the mechanisms of interaction between tumour cells and host immune response may allow the introduction of new, effective therapies that are not as toxic as conventional methods of cancer treatment. Despite the evidence of anti-tumour responses including cytotoxic T-lymphocyte response to antigens of the tumour - the host immune system is frequently unable to eliminate the neoplastic clone.

The main mechanisms of tumour escape from host immune system are: changes in the expression of antigens and costimulatory molecules, direct suppression of the function of dendritic cells, T cell cytokine production, and the induction of regulatory $\mathrm{T}$ cells (Treg) that have the ability to inhibit the immune response $[1,2]$. Tumour microenvironment (TME) may also activate cells other than Treg; over the past few years a new member of the tumour-host interacting cells has been characterized, i.e. suppressor cells derived from myeloid progenitors (myeloid-derived suppressor cells - MDSCs). This population was detected at the end of the $20^{\text {th }}$ century, but its final name was established in 2007 [3]. Previous terms such as immature myeloid cells (IMC) and myeloid suppressor cells (MSC) did not accurately reflect their origin and function. MDSCs are a heterogeneous population of cells derived from myeloid lineage, consisting of immature macrophages, granulocytes, dendritic cells, and other cells in the early stages of differentiation, which are potent immunosuppressants. The presence of MDSCs was established in animal models of cancer as well as in humans $[4,5]$. The described cells were initially found in the spleen, bone marrow, and tumour microenvironment and recently - even in the peripheral blood. This population inhibits immune response, including tumour-associated antigens (TAA). The accumulation of these cells also occurs in conditions such as chronic inflammation (including bacterial and parasitic infections), injuries, and graft versus host disease after transplantation of haematopoietic cells [6-9]. In the view of the above, this seems to be a promising approach to block MDSC trafficking and infiltration and therefore reduce suppressive activities of MDSCs in the discussed disorders [10,11].

\section{Characteristics of myeloid-derived suppressor cells}

MDSCs are known as a heterogeneous cell population consisting of myeloid cells in various stages of dif- 
ferentiation without mature myeloid-associate markers on their surface $[3,12]$. In murine model tumour, MDSCs were defined as cells with co-expression of CD11b and Gr1 markers; however, a lack of human homologue of Gr1 caused various problems with their immunophenotyping, highlighted in scientific literature [13]. Human MDSCs are less understood but are mostly introduced as immature myeloid cells with surface expression of CD33+ consisting of dendritic cell, macrophage, and granulocyte progenitors [14]. The main function of MDSCs was a generalised suppressive impact on the immune response, predominantly through direct inhibition of the cytotoxic functions of T cells and NK cells [15]. As MDSCs arise from myeloid progenitor cells without the potential of further differentiation into mature dendritic cells, granulocytes, or macrophages, they can be divided into three important phenotypes: granulocytic, promyelocytic, and monocytic [16]. Diversification between monocytic and granulocytic (polymorphonuclear) subsets is based on specific markers $\left(\mathrm{CD} 11 \mathrm{~b}^{+} \mathrm{Ly}_{6 \mathrm{C}^{\text {high }}}\right.$ Ly6G-) typical of monocytes M-MDSCs and different phenotypes $\left(\mathrm{CD} 11 \mathrm{~b}^{+} \mathrm{Ly} 6 \mathrm{C}^{\text {low }} \mathrm{Ly} 6 \mathrm{G}^{+}\right)$common in granulocytes G-MDSCs division $[17,18]$. Additionally, this group can express granulocytic marker CD15+ or CD66b+ [19]. There were found also functional differences between each of the subsets: granulocytic MDSCs are able to produce higher levels of arginase and have been shown to generate higher levels of reactive oxygen species (ROS) [20, 21]. This is one of the mechanisms by which granulocytic MDSCs are able to suppress T cells inducing T-cell apoptosis. In addition ROS can block activation of T-cell receptor (TCR) by its nitrosylation, and as a result inhibit binding of an antigen. Monocytic MDSCs can express arginase and inducible nitric oxide synthetase (iNOS); however, they do not produce high levels of ROS [19, 22-25].

\section{MDSCs in tumour microenvironment}

Initial studies concerning MDSCs started in the 1990s while on experiments with anti-cancer vaccinations [26]. Further research revealed MDSCs as cells with an exceptional ability to suppress both innate and adaptive immune responses while stimulating tumour angiogenesis, neoplasm invasion, and metastasis [27, 28]. Immature myeloid cells are continually produced in the bone marrow of healthy individuals, and then they differentiate into mature form without causing observable immunosuppression. Normal myeloid cell differentiation is disrupted within cancer environment [29, 30]. Tumour-derived stromal cells are able to release multiple cytokines interfering with the myeloid compartment. A wide range of colony stimulating factors such as GM-CSF, G-CSF, M-CSF, SCF as well as VEGF and IL-3 promote myelopoiesis and partially inhibit myeloid cell maturation [13, 31-34]. Furthermore, pro-inflammatory cytokines - IL-1 $\beta$, IL-6, and $\mathrm{PGE}_{2}$ - induce myeloid differentiation towards immunosuppressive
MDSCs; IFN- $\gamma$ along with LPS promotes their multiplying, and then TGF- $\beta$ impacts on their concentration. Chronic inflammation associated with tumour development is promoted by several mechanisms involved in the production of proangiogenic factors, matrix metalloproteinases (MMPs), and damage-associated molecular pattern molecules (DAMPs). All of the above leads to MDSC accumulation and MDSC suppressive effects [12, 35-39].

Several suppressive mechanisms attributed to MDSCs in tumour environment have been proved so far (Fig. 1). By limiting the amino acids such as L-Arg, L-Trp, and L-Cys, they are able to inhibit T-cell activation as well as proliferation $[12,40]$. One of the well-defined mechanisms is upregulation of nitric oxide synthase 2 (NOS2), which generates nitric oxide (NO), ROS, and peroxynitrate. Production of NO (nitric oxide) prevents IL-2 signalling, thereby impairing the proliferation of cytotoxic and memory $\mathrm{T}$ cells. Secretion of reactive oxygen species (ROS) inhibits peptide detection by $\mathrm{T}$ cells and prompts $\mathrm{T}$-cell apoptosis. The increased peroxynitrate accumulation leads to the nitration of the CD8 TCR with inhibition of CD8+ T-cell activity [41-43]. Downregulation of L-selectin and E-selectin impairs T-cell migration to lymph nodes and the tumour region [44]. Cell-to-cell contact between MDSCs and macrophages as well as cross-talks with dendritic cells (DCs) in tumour-bearing environment promote metastasis and impair anti-tumour function of both cell classes [45]. Also, inhibition of NK function by MDSCs by deactivating NKG2D receptor was revealed. Although MDSCs play an important role in tumour progression, the specific signals triggering the accumulation of MDSCs in cancer patients remain unidentified $[46,47]$.

\section{Gynaecological oncology and MDSCs}

Incidence of breast cancer and female reproductive system neoplasms is continuously increasing [48]. Worldwide, breast and ovarian cancers account for $26.6 \%$ (2008) of all cancer cases among females [49]. Breast cancer is the most common cancer among females, with an estimated 249,260 new cases in 2016, while ovarian cancer is responsible for $5 \%$ of all oncological deaths and its mortality rate is higher than of any other gynaecological cancer [ 48 , 50, 51]. Among gynaecological cancers, ovarian cancer is the third most frequent, next to cervix uteri and corpus uteri cancers [48]. Although previous decades contributed to major progress in targeted therapy of many malignancies, the treatment of gynaecological cancers still remains a challenging task [52].

Recent studies have revealed many facts considering gynaecological tumour microenvironment and the critical role of MDSCs in the immune network [53]. Though most studies focus on ovarian cancer, the incidence of MDSCs correlating with abnormal arginase- 1 activity has been confirmed so far in endometrial cancer and uterine sarco- 


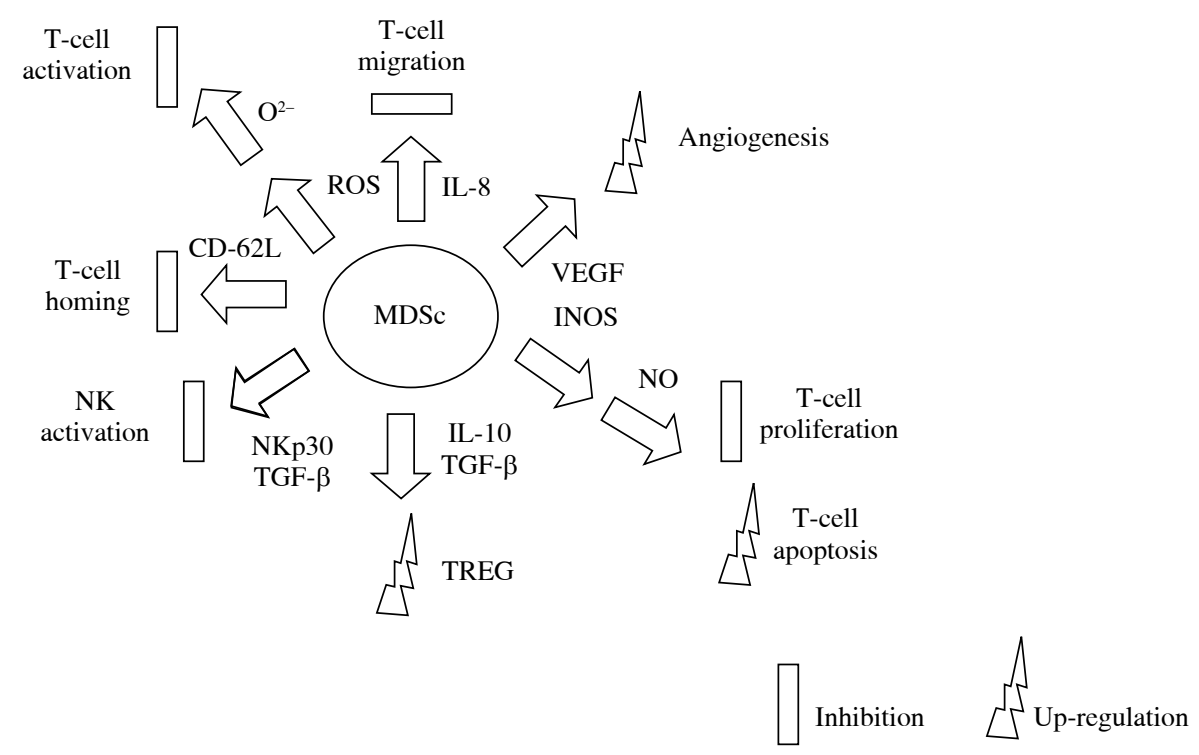

Fig. 1. The role of MDSCs in the suppression of anti-cancer cellular response and tumour growth

ma [54]. In another type of cancer of the female reproductive tract, cervical cancer, the increased accumulation of MDSCs was induced by tumour-derived G-CSF. G-CSF expression was proven to be an independent poor prognostic factor in cervical cancer patients treated with platinum-based chemotherapy. Moreover, MDSC incidence was responsible for the development of cisplatin resistance in G-CSF-producing cervical cancer [55].

\section{MDSC characteristics in ovarian cancer}

Epithelial ovarian cancer (EOC) consists of a range of specific histological subtypes. About $70 \%$ of cases of EOC are serous carcinoma. Other subtypes are endometrioid, mucinous, and clear cell, which are associated with a worse prognosis than all the other subtypes [56, 57]. A recent study suggests that EOC can be divided into two subtypes based on IL-6, IL-6R, and immune infiltration. The first group comprises tumours with high expression of IL-6R and low infiltration by mature myeloid cells, with good patient survival, suggesting that determination of IL-6R expression might be useful as a prognostic marker. The second group covers tumours without expression of IL-6R, but with a high level of IL-6, and infiltrated with mature CD163+ myeloid cells [58].

EOC is typically diagnosed at advanced stages. The percentage of five-year survival is approx. $20-25 \%$ for tumours in clinical stage III according to the International Federation of Gynaecology and Obstetrics (FIGO), and only $5 \%$ for stage IV disease, which confirms the great variability in progression-free survival rates and overall survival among patients with advanced EOC [59]. The causes of such a high rate of mortality seem to be related to the complex biology as well as the huge heterogeneity of EOC [60]. Typical treatment - surgical removal of the tumour with additional chemotherapy - is not efficient and leads in the vast majority of cases to progression of the disease [61]. EOC is characterised clinically by ascites and peritoneal implants; molecularly by accumulation of tumour-associated macrophages and MDSCs, which have been suggested since the 1990s as a critical immunosuppressive component in tumour microenvironment [62-64].

\section{Mechanisms guiding MDSCs to human cancer environment}

The mechanism inducing MDSCs into TME of EOC is poorly understood. Several studies have suggested a variety of mechanisms of MDSC induction among tumour cell lines, highlighting role of each tumour type's specific combination of inflammatory cytokines. Prostaglandin $\mathrm{E}_{2}$ $\left(\mathrm{PGE}_{2}\right)$ is described specifically for EOC as an inducing factor of CXCR4-CXCL12 axis. It is a possible pathway mediating the attraction of monocytic MDSCs into the TME of ovarian cancer patients. CXCL12, known as stromal cell-derived factor-1, is a well-defined molecule enhancing tumour growth, migration, and metastasis, produced by stromal and tumour cells of TME. CXCL12 and its receptor CXCR4 are greatly involved in cancer progression by direct activation of cancer cells as well as induction of angiogenesis, Treg, and DCs into the tumour surrounding. In peritoneal fluid isolated from EOC patients, both CXCL12 and CXCR4 are controlled by the tumour-associated inflammatory mediator $-\mathrm{PGE}_{2}$, which attracts MDSCs into the ascites microenvironment. $\mathrm{PGE}_{2}$ was essential both for expression of functional CXCR4 in can- 
cer-associated MDSCs and for production of its ligand CXCL12 [33, 65-67]. In contrast to the above, Lechner et al. indicate that the addition of cyclooxygenase 2 (COX2 - the key enzyme in prostaglandin $\mathrm{PGE}_{2}$ synthesis) inhibitors to ovarian and cervical tumour cell line-PBMC co-cultures did not significantly decrease MDSC induction, which strongly suggests the existence of another mechanism inducing MDSCs in ovarian TME [14]. Interestingly, recent data have revealed that IFN- $\gamma$ and TNF- $\alpha$ synergise in the induction of COX2 and subsequently hyperactivate MDSCs within the TME of ovarian cancer patients. A new mechanism focuses on the synergistic action of IFN- $\gamma$ and TNF- $\alpha$, which could not be achieved by either of these factors alone [68]. IL-6 was the cytokine involved in another possible way of induction of MDSCs in TME of ovarian cancer. IL-6 is a major mediator of cancer-related inflammation, tumour growth, tumour angiogenesis, and tumour myeloid cell infiltration in ovarian cancer. Differentiation of MDSCs can be mediated by IL-6 [3, 12, 15, 58]. Reinartz et al. defined a subgroup of ovarian cancer patients with a poor clinical outcome, with high CD163 expression (myeloid cells marker) and high IL-6 levels in ascites [69]. In an experimental study Wouters et al. indicate IL-6 and its ligand IL-6R as opposite markers for tumour-infiltrating myeloid cell infiltration and survival. Tumours with a high expression of IL-6R displayed low mature myeloid cell infiltration and a longer disease-specific survival. In contrast, tumours with high epithelial IL-6 expression displayed a dense infiltration of tumour-infiltrating myeloid and were correlated with a shorter survival [58].

\section{MDSCs influence to ovarian cancer progression}

Initially MDSCs in ovarian tumour environment were described in 2004 as vascular leukocytes (VLCs); the population of ovarian tumour-associated leukocytes of unknown origin and surface markers such as F4/80 and CD11b [70, 71]. The presence of VLCs highly inhibited CD8+ and CD4+ T-cell activity with IFN- $\gamma$ release by $>95 \%$. Closer inspection revealed VLC dependence on ARG1 enzyme, and that inhibition with the ARG1-specific inhibitor nor-NOHA restores activation of CD8+ and CD4+ T cells. Additionally, production of ARG-1 results in generation of ROS. The subsequent characterisation of the tumour-associated leukocytes in ovarian cancer provided insight into the phenotype referred to as MDSC [72]. Recently published research has confirmed the "stemness" of MDSCs existing in the ovarian cancer environment. This highlights the importance of interactions between MDSCs and cancer stem cells via the MDSC-microRNA101-CtBP2 network, which may affect the tumour's phenotype and the patient's outcome. MDSCs trigger miRNA101 expression in cancer cells. Therefore, miRNA101 influences the corepressor gene C-terminal binding protein-2 (CtBP2), and
CtBP2 directly targeted stem cell core genes resulting in increased cancer cell stemness and increased metastatic and tumorigenic potential [73].

Further studies have implicated the importance of oxidative stress in creating immunosuppressive TME in EOC. Activated monocytes, neutrophils, and MDSCs have started to be considered as the components of the chronic inflammatory environment that suppresses T-cell function. On the one hand, previous studies revealed that ROS generation is one of the main characteristics of MDSCs from tumour-bearing mice, and on the other hand ROS's ability in promoting MDSCs development and/or immunosuppressive activity was mentioned. NADPH oxidase (NOX2), a major source of ROS in activated phagocytes, potentially has multiple effects on the tumour microenvironment that either promotes or inhibits tumour progression, including modulation of the cytokine's influence, inflammatory cell recruitment, and antigen display and cross-presentation [74-77]. However, a recently published experimental study has not indicated NADPH oxidase as a factor of tumour progression in murine EOC. The accumulation of MDSCs locally and systemically was similar in genetically engineered NADPH oxidase-deficient mice in comparison to wild type mice. The suppressive effect of MDSCs on stimulated T-cell proliferation was NADPH oxidase-independent. Although MDSC retention and immunosuppression in murine EOC is NADPH oxidase-independent, the lack of effect of NOX2 on MDSC accumulation and function does not rule out an effect of other sources of ROS [78].

\section{Future therapy}

Although new multiple mechanisms of ovarian tumour network are being constantly revealed, a potential target of the immunotherapy is still absent. Recently published studies highlighted that essential components of future therapeutic strategies should include combination treatments aimed at dealing with the complement inhibitors, together with accurate patient selection. Interestingly, the latest achievement regarding future therapy of MDSCs in ovarian cancer is not only targeted therapy [79]. The efficiency of conventional chemotherapy can be increased by using aptamer-A8, which blocks heat shock protein HSP70 on exosomes. This molecule is able to activate MDSCs via two toll-like receptors on their surface. The amount of this molecule is highly increased by chemotherapeutic agents such as cisplatin or 5-fluorouracil, and it is correlated with higher activation of MDSCs. This mechanism was not observed if chemotherapeutics were combined with $\mathrm{A} 8$, which strongly potentiated the antitumor effect of the drugs [80]. Another interesting feature of TME in EOC is correlation between Th17 and MDSC-associated NOS2 and MDSC-produced exogenous NO. The development of human Th17 cells from naive, effector, and memory CD4+ T-cell precursors is induced by the previously identified Th17-driving cytokines (IL-1 $\beta$, 
IL-6, and IL-23) or by IL-1 $\beta / \mathrm{IL}-6 / \mathrm{IL}-23$-producing MDSCs. Th17 increase is promoted by NO produced by human MDSCs and mainly depends on the induction of endogenous NOS2 in differentiating CD4+ T cells [81-83].

\section{The authors declare no conflict of interest.}

\section{References}

1. Kebelmann-Betzing C, Korner G, Badiali L, et al. (2001): Characterization of cytokine, growth factor receptor, costimulatory and adhesion molecule expression patterns of bone marrow blasts in relapsed childhood B cell precursor ALL. Cytokine 13: 39-50.

2. Łuczyński W, Stasiak-Barmuta A, Iłendo E, et al. (2006): Low expression of costimulatory molecules and mRNA for cytokines are important mechanisms of immunosuppression in acute lymphoblastic leukemia in children? Neoplasma 53: 301-304.

3. Gabrilovich DI, Bronte V, Chen SH, et al. (2007): The terminology issue for myeloid-derived suppressor cells. Cancer Res 67: 425-426.

4. Bronte V, Apolloni E, Cabrelle A, et al. (2000): Identification of $\mathrm{CD} 11 \mathrm{~b}+/ \mathrm{Gr}-1+/ \mathrm{CD} 31+$ myeloid progenitor capable of activating or suppressing CD8+ T cells. Blood 96: 3838-3846.

5. Zea AH, Rodriquez PC, Atkins MB, et al. (2005): Arginaseproducing myeloid suppressor cells in renal cell carcinoma patients: a mechanism of tumor evasion. Cancer Res 65: 3044-3048.

6. Billiau AD, Fevery S, Rutgeerts O, et al. (2003): Transient expansion of Mac1+Ly6-G+Ly6-C+ early myeloid cells with suppressor activity in spleens of murine radiation marrow chimeras: possible implications for the graft-versus-host and graft-versus-leukemia reactivity of donor lymphocyte infusions. Blood 102: 740-748.

7. Bunt SK, Sinha P, Clements VK, et al. (2006): Inflammation induces myeloid-derived suppressor cells that facilitate tumor progression. J Immunol 176: 284-290.

8. Zhang Q, Fujino M, Xu J, Li X (2015): The Role and Potential Therapeutic Application of Myeloid-Derived Suppressor Cells in Allo- and Autoimmunity. Mediators Inflamm 2015: 421927.

9. Lai D, Qin C, Shu Q (2014): Myeloid-Derived Suppressor Cells in Sepsis. Biomed Res Int 2014: 598654

10. Katoh H, Watanabe M (2015): Myeloid-Derived Suppressor Cells and Therapeutic Strategies in Cancer. Mediators Inflamm 2015: 159269.

11. Vetsika EK, Koinis F, Gioulbasani M, et al. (2014): A circulating subpopulation of monocytic myeloid-derived suppressor cells as an independent prognostic/predictive factor in untreated non-small lung cancer patients. J Immunol Res 2014: 659294.

12. Gabrilovich DI, Ostrand-Rosenberg S, Bronte V (2012): Coordinated regulation of myeloid cells by tumours. Nat Rev Immunol 12: 253-268.

13. Dolcetti L, Peranzoni E, Ugel S, et al. (2010): Hierarchy of immunosuppressive strength among myeloid-derived suppressor cell subsets is determined by GM-CSF. Eur J Immunol 40: $22-35$

14. Lechner MG, Megiel C, Russell SM, et al. (2011): Functional characterization of human $\mathrm{Cd} 33+$ and $\mathrm{Cd} 11 \mathrm{~b}+$ myeloid-derived suppressor cell subsets induced from peripheral blood mononuclear cells co-cultured with a diverse set of human tumor cell lines. J Translat Med 9: 90.

15. Kapka-Skrzypczak L, Wolinska E, Szparecki G, et al. (2015): The immunohistochemical analysis of membrane-bound CD55, CD59 and fluid-phase FH and FH-like complement inhibitors in cancers of ovary and corpus uteri origin. Cent Eur J Immunol 40: 349-353.

16. Youn JI, Nagaraj S, Collazo M, Gabrilovich D (2008): Subsets of myeloid-derived suppressor cells in tumor-bearing mice. J Immunol 181: 5791-5802.

17. Ning G, She L, Lu L, et al. (2015): Analysis of Monocytic and Granulocytic Myeloid-Derived Suppressor Cells Subsets in Patients with Hepatitis C Virus Infection and Their Clinical Significance. Biomed Res Int 2015: 385378.

18. Movahedi K, Guilliams M, Van den Bossche J, et al. (2008): Identification of discrete tumor-induced myeloid-derived suppressor cell subpopulations with distinct T cell-suppressive activity. Blood 111: 4233-4244.

19. Dumitru CA, Moses K, Trellakis S, et al. (2012): Neutrophils and granulocytic myeloid-derived suppressor cells: Immunophenotyping, cell biology and clinical relevance in human oncology. Cancer Immunol Immunother 61: 1155-1167.

20. Gabrilovich DI, Nagaraj S (2009): Myeloid-derived suppressor cells as regulators of the immune system. Nat Rev Immunol 9: 162-174.

21. Rodríguez PC, Ochoa AC (2008): Arginine regulation by myeloid derived suppressor cells and tolerance in cancer: mechanisms and therapeutic perspectives. Immunological reviews. Immunol Rev 222: 180-191.

22. Pan PY, Ma G, Weber KJ, et al. (2010): Immune stimulatory receptor CD40 is required for T-cell suppression and $\mathrm{T}$ regulatory cell activation mediated by myeloid-derived suppressor cells in cancer. Cancer Res 70: 99-108.

23. Malutan AM, Drugan T, Costin N, et al. (2015): Pro-inflammatory cytokines for evaluation of inflammatory status in endometriosis. Centr Eur J Immunol 40: 96-102.

24. Kusmartsev SA, Li Y, Chen SH (2000): Gr-1+ myeloid cells derived from tumor-bearing mice inhibit primary $\mathrm{T}$ cell activation induced through CD3/CD28 costimulation. J Immunol 165: 779-785.

25. Yazan S K, Basil JA, Elkord E (2014): Increased Levels of Granulocytic Myeloid-Derived Suppressor Cells in Peripheral Blood and Tumour Tissue of Pancreatic Cancer Patients. J Immunol Res 2014: 879897.

26. Garrity T, Pandit R, Wright MA, et al. (1997): Increased presence of CD34+ cells in the peripheral blood of head and neck cancer patients and their differentiation into dendritic cells. Int J Cancer 73: 663-669.

27. Marx J (2008): Cancer immunology. Cancer's bulwark against immune attack: MDS cells. Science 319: 154-156.

28. Ostrand-Rosenberg S, Sinha P (2009): Myeloid-Derived Suppressor Cells: Linking Inflammation and Cancer. J Immunol 182: 4499-4506.

29. Melani C, Chiodoni C, Forni G, Colombo MP (2003): Myeloid cell expansion elicited by the progression of spontaneous mammary carcinomas in c-erbB-2 transgenic BALB/c mice suppresses immune reactivity. Blood 102: 2138-2145.

30. Quail D, Joyce J (2013): Microenvironmental regulation of tumor progression and metastasis. Nat Med 19: 1423-1437.

31. Kusmartsev S, Su Z, Heiser A, Dannull J (2008): Reversal of myeloid cell-mediated immunosuppression in patients with metastatic renal cell carcinoma. Clin Cancer Res 14: 82708278 . 
32. Melani C, Chiodoni C, Forni G, Colombo MP (2003): Myeloid cell expansion elicited by the progression of spontaneous mammary carcinomas in c-erbB-2 transgenic BALB/c mice suppresses immune reactivity. Blood 102: 2138-2145.

33. Kusmartsev S, Eruslanov E, Kubler H, et al. (2008): Oxidative stress regulates expression of VEGFR1 in myeloid cells: link to tumor-induced immune suppression in renal cell carcinoma. J Immunol 181: 346-353.

34. Serafini P, De Santo C, Marigo I, et al. (2004): Derangement of immune responses by myeloid suppressor cells. Cancer Immunol Immunother 53: 64-72.

35. Eliopoulos AG, Dumitru CD, Wang CC, et al. (2002): Induction of COX-2 by LPS in macrophages is regulated by Tpl2-dependent CREB activation signals. EMBO J 21: 4831-4840.

36. Sinha P, Clements VK, Ostrand-Rosenberg S (2005): Reduction of myeloid-derived suppressor cells and induction of M1 macrophages facilitate the rejection of established metastatic disease. J Immunol 174: 636-645.

37. Trikha P, Carson WE (2014): Signaling pathways involved in MDSC regulation. Biochimica et Biophysica Acta 1846: 55-65.

38. Wesolowski R, Markowitz J, Carson WE (2013): Myeloid derived suppressor cells - a new therapeutic target in the treatment of cancer. J Immunotherapy Cancer 1: 10.

39. Shi Q, Cheng L, Liu Z, et al. (2015): The p38 MAPK inhibitor SB203580 differentially modulates LPS-induced interleukin 6 expression in macrophages. Cent Eur J Immunol 40: 276-282.

40. Parker KH, Beury DW, Ostrand-Rosenberg S (2015): Myeloid-Derived Suppressor Cells: Critical Cells Driving Immune Suppression in the Tumor Microenvironment. Adv Cancer Res 128: 95-139.

41. Kesarwani P, Murali AK, Al-Khami AA, Mehrotra S (2013): Redox Regulation of T-Cell Function: From Molecular Mechanisms to Significance in Human Health and Disease. Antioxid Redox Signal 18: 1497-1534.

42. Giallongo C, Parrinello N, Brundo MV, et al. (2015): Myeloid Derived Suppressor Cells in Chronic Myeloid Leukemia. Front Oncol 5: 107.

43. Mazzoni A, Bronte V, Visintin A, et al. (2002): Myeloid suppressor lines inhibit $\mathrm{T}$ cell responses by an NO-dependent mechanism. J Immunol 168: 689-695.

44. Hanson EM, Clements VK, Sinha P, et al. (2009): Myeloid-derived suppressor cells down-regulate 1-selectin expression on CD4+ and CD8+ T cells. J Immunol 183: 937-944.

45. Ostrand-Rosenberg S, Sinha P, Beury DW, Clements VK (2012): Cross-talk between myeloid-derived suppressor cells (MDSC), macrophages, and dendritic cells enhances tumor-induced immune suppression. Semin Cancer Biol 22: 275-281.

46. Li H, Han Y, Guo Q, et al. (2009): Cancer-expanded myeloid-derived suppressor cells induce anergy of NK cells through membrane-bound TGF-beta 1. J Immunol 182: 240-249.

47. Brandau S, Moses K, Lang S (2013): The kinship of neutrophils and granulocytic myeloid-derived suppressor cells in cancer: cousins, siblings or twins? Semin Cancer Biol 23: 171-182.

48. Siegel RL, Miller KD, Jemal A (2016): Cancer statistics, 2016. CA Cancer J Clin 66: 7-30.

49. Ferlay J, Shin HR, Bray F, et al. (2010): Estimates of worldwide burden of cancer in 2008: GLOBOCAN. Int J Cancer 127: 2893-2917.

50. Navaneelan T (2015): Trends in the incidence and mortality of female reproductive system cancers. Health at a Glance. Statistics Canada catalogue no. 82-624-X.
51. Permuth-Wey J, Sellers TA (2009): Epidemiology of ovarian cancer. Methods Mol Biol 472: 413-437.

52. Sawyers C (2004): Targeted cancer therapy. Nature 432: 294297.

53. Diaz-Montero CM, Finke J, Montero AJ (2014): Myeloid-derived suppressor cells in cancer: therapeutic, predictive, and prognostic implications. Semin Oncol 41: 174-184.

54. Vanderstraeten A, Luyten C, Verbist G, et al. (2014): Mapping the immunosuppressive environment in uterine tumors: implications for immunotherapy. Cancer Immunol Immunother 63: 545-557.

55. Kawano M, Mabuchi S, Matsumoto Y, et al. (2015): The significance of G-CSF expression and myeloid-derived suppressor cells in the chemoresistance of uterine cervical cancer. Sci Rep 5: 18217.

56. Clark TG, Stewart ME, Altman DG, et al. (2001): A prognostic model for ovarian cancer. Br J Cancer 85: 944-952.

57. Agarwal R, Kaye SB (2005): Prognostic factors in ovarian cancer: how close are we to a complete picture? Ann Oncol 16: 4-6.

58. Wouters M, Dijkgraaf E, Kuijjer M, et al. (2014): Interleukin-6 receptor and its ligand interleukin-6 are opposite markers for survival and infiltration with mature myeloid cells in ovarian cancer. Oncoimmunology 12: e962397.

59. Makar AP, Baekelandt M, Tropé CG, Kristensen GB (1995): The prognostic significance of residual disease, FIGO substage, tumor histology, and grade in patients with FIGO stage III ovarian cancer. Gynecol Oncol 56: 175-180.

60. Solito S, Marigo I, Pinton L, et al. (2014): Myeloid-derived suppressor cell heterogeneity in human cancers. Ann N Y Acad Sci 1319: 47-65.

61. Charbonneau B, Goode EL, Kalli KR, et al. (2013): The immune system in the pathogenesis of ovarian cancer. Crit Rev Immunol 33: 137-164.

62. Ugel S, De Sanctis F, Mandruzzato S, Bronte V (2015): Tumor-induced myeloid deviation: when myeloid-derived suppressor cells meet tumor-associated macrophages. J Clin Invest 125: 3365-3376.

63. Lavoué V, Thédrez A, Levěque J, et al. (2013): Immunity of human epithelial ovarian carcinoma: the paradigm of immune suppression in cancer. J Transl Med 11: 147.

64. Wertel I, Nowicka A, Rogala E, Kotarski J (2011): Peritoneal immune system in patients with advance epithelial ovarian cancer. Int Rev Immunol 30: 87-101.

65. Obermajer N, Muthuswamy R, Odunsi K, et al. (2011): PGE2-induced CXCL12 production and CXCR4 expression controls the accumulation of human MDSCs in ovarian cancer environment. Cancer Res 71: 7463-7470.

66. Eruslanov E, Neuberger M, Daurkin I, et al. (2011): Circulating and tumor-infiltrating myeloid cell subsets in patients with bladder cancer. Int J Cancer 130: 1109-1119.

67. Kajiyama H, Shibata K, Terauchi M, et al. (2008): Involvement of SDF-1alpha/CXCR4 axis in the enhanced peritoneal metastasis of epithelial ovarian carcinoma. Int J Cancer 122: 91-99.

68. Wong JL, Obermajer N, Odunsi K, et al. (2016): Synergistic COX2 Induction by IFN $\gamma$ and TNF $\alpha$ Self-Limits Type-1 Immunity in the Human Tumor Microenvironment. Cancer Immunol Res 4: 303-311.

69. Reinartz S, Schumann T, Finkernagel F, et al. (2014): Mixed-polarization phenotype of ascites-associated macrophages in human ovarian carcinoma: correlation of CD163 expression, cytokine levels and early relapse. Int J Cancer 134: 32-42. 
70. Conejo-Garcia JR, Benencia F, Courreges MC, et al. (2004): Tumor-infiltrating dendritic cell precursors recruited by a beta-defensin contribute to vasculogenesis under the influence of Vegf-A. Nat Med 10: 950-958.

71. Conejo-Garcia JR, Buckanovich RJ, Benencia F, et al. (2005): Vascular leukocytes contribute to tumor vascularization. Blood 105: 679-681.

72. Bak SP, Alonso A, Jo Turk M, Berwin B (2008): Murine ovarian cancer vascular leukcoytes require murine ovarian cancer require arginasa- 1 activity for T-cell suppression. Mol Immunol 46: 258-268.

73. Cui TX, Kryczek I, Zhao L, et al. (2013): Myeloid derived suppressor cells enhance stemness of cancer cells by inducing microRNA101 and suppressing the corepressor CtBP2. Immunity 39: 611-621.

74. Savina A, Jancic C, Hugues S, et al. (2006): NOX2 controls phagosomal $\mathrm{pH}$ to regulate antigen processing during crosspresentation by dendritic cells. Cell 126: 205-218.

75. Jancic C, Savina A, Wasmeier C, et al. (2007): Rab27a regulates phagosomal $\mathrm{pH}$ and $\mathrm{NADPH}$ oxidase recruitment to dendritic cell phagosomes. Nat Cell Biol 9: 367-378.

76. Segal BH, Han W, Bushey JJ, et al. (2010): NADPH oxidase limits innate immune responses in the lungs in mice. PLoS One 5: e9631.

77. Romani L, Fallarino F, De Luca A, et al. (2008): Defective tryptophan catabolism underlies inflammation in mouse chronic granulomatous disease. Nature 451: 211-215.

78. Godoy HE, Khan AN, Vethanayagam RR, et al. (2013): Myeloid-Derived Suppressor Cells Modulate Immune Responses Independently of NADPH Oxidase in the Ovarian Tumor Microenvironment in Mice. PLoS One 8: e69631.74.

79. Kubatova A, Erdem A, Erdem M, et al. (2015): Serum cytokine and growth factor levels in patients with endometriosis. Centr Eur J Immunol 38: 500-504.

80. Gobbo J, Marcion G, Cordonnier M, et al. (2015): Restoring Anticancer Immune Response by Targeting Tumor-Derived Exosomes With a HSP70 Peptide Aptamer. J Natl Cancer Inst 108: 330.

81. Rieber N, Wecker I, Neri D, et al. (2014): Extracorporeal photopheresis increases neutrophilic myeloid-derived suppressor cells in patients with GvHD. Bone Marrow Transplant 49: 545-552.

82. Obermajer N, Wong JL, Edwards RP, et al. (2013): Induction and stability of human Th17 cells require endogenous NOS2 and cGMP-dependent NO signaling. J Exp Med 210: 14331445.

83. Baharlou R, Atashzar MR, Vasmehjani AA, et al. (2016): Reduced levels of T-helper 17-associated cytokines in the serum of patients with breast cancer: indicators for following the course of disease. Centr Eur J Immunol 41: 78-85. 